\title{
Measurement of Tryptophan Metabolites in Healthy Old Men and Patients of Type 2 Diabetes Mellitus (T2DM)
}

\author{
Akikazu Takada1 ${ }^{*}$, Fumiko Shimizu², Tetsuya Takao², Junichi Masuda ${ }^{3}$ \\ ${ }^{1}$ International Projects on Food and Health (NPO), Tokyo, Japan \\ ${ }^{2}$ Faculty of Life and Environmental Sciences, Showa Women's University, Tokyo, Japan \\ ${ }^{3}$ Global Application Development Center, Shimadzu Corporation, Kyoto, Japan \\ Email: *takadaa@mwd.biglobe.ne.jp
}

How to cite this paper: Takada, A., Shimizu, F., Takao, T. and Masuda, J. (2018) Measurement of Tryptophan Metabolites in Healthy Old Men and Patients of Type 2 Diabetes Mellitus (T2DM). Food and $\mathrm{Nu}$ trition Sciences, 9, 1206-1220.

https://doi.org/10.4236/fns.2018.910087

Received: September 25, 2018

Accepted: October 21, 2018

Published: October 24, 2018

Copyright $\odot 2018$ by authors and Scientific Research Publishing Inc. This work is licensed under the Creative Commons Attribution International License (CC BY 4.0).

http://creativecommons.org/licenses/by/4.0/

\begin{abstract}
Background: Plasma levels of tryptophan (TRP) metabolites have not been measured extensively in patients of type 2 diabetes mellitus (T2DM). Methods: Metabolites analysis was performed by a liquid chromatograph tandem mass spectrometer, the LCMS-8060 quadrupole mass spectrometer combined with Nexera X2 liquid chromatograph system (Shimadzu Corporation, Kyoto, Japan). Body mass index (BMI) and TRP metabolites have been measured in healthy old men $(n=20)$ and patients of T2DM $(n=20)$. TRP metabolites were measured by using the ultrahigh speed liquid chromatography-mass spectroscopy (Shimadzu Corporation). Results: The plasma levels of 5-hydroxytryptophan (5-HTRP), 5-hydroxyindoleacetic acid (5-HIAA), kynurenic acid (KNA), 3-hydroxykynurenine (3-HKN), and 3-hydroxyanthranilic acid (3-HAA) were higher in patients of T2DM than healthy old men. Since 5HTRP and 5-HIAA belong to the serotonin pathway, and KNA, 3-HKN, and 3-HAA belong to the KN pathway of TRP metabolism, these pathways were activated more in the patients of T2DM. Since plasma levels of Indole-3-acetic acid were not elevated in T2DM, that pathway was not activated more in T2DM. Serotonin levels were not increased but 5-HIAA levels were increased in the plasma of T2DM patients, which may mean that serotonin was quickly metabolized to 5-HIAA in the patients of T2DM. Conclusion: Plasma levels of tryptophan metabolites in serotonin and kynurenine pathways increased in T2DM patients. Obesity expressed by BMI may not influence tryptophan metabolism in healthy old men and T2DM patients. These results indicate that our new method of the simultaneous measurements of all the tryptophan metabolites is the powerful measure to identify factor related to endogenous stresses seen in DM.
\end{abstract}




\section{Keywords}

Tryptophan, Serotonin, 5-Hydroxyindole Acetic Acid, Kynurenine, 3-Hydroxykynurenine, Kynurenic Acid

\section{Introduction}

Tryptophan (TRP) is one of essential amino acids for protein synthesis, but it also serves as a substrate for the generation of several bioactive compounds with important physiological roles. TRP is converted to serotonin (5-hydroxytryptamine), an important neurotransmitter involved in the control of many responses in the central nervous system (CNS) and linked to alterations in mood, anxiety, or cognition [1]. Serotonin can be further converted to $\mathrm{N}$-acetylserotonin (NAS) and melatonin, influencing control over circadian rhythmicity to the list of biological roles for TRP metabolites [2]. It is known that in mammals, the majority of free TRP is degraded through the kynurenine pathway (KP) and generates many metabolites involved in inflammation, immune response, and excitatory neurotransmission [3].

We have shown that foot shock applied to rats resulted in increase in not only brain serotonin levels but kynurenine in plasma, kidney, liver and every part of the brain [4] [5] [6].

Several TRP metabolites have been shown to exhibit neuroexcitatory, convulsant, and toxic properties [7] [8]

In the periphery, only $1 \%$ of dietary TRP was converted to serotonin and more than $95 \%$ was metabolized to kynurenines [9] [10].

We thought that TRP metabolism may have been changed in patients of T2DM because patents are considered to get various stresses both physiological and psychological including self-care. So we measured plasma level of TRP metabolites, and showed that TRP metabolites increased in plasma of patients of type 2 diabetes mellitus (T2DM) [11].

In the present paper, we report in details of the methodology of the simultaneous measurement of TRP metabolites in healthy old men and patients of T2DM.

\section{Ethics}

This work has been approved by the Ethical committees of Showa Women's University and NPO (non-profit organization) "International projects on food and health" and has been carried out in accordance with The Code of Ethics of the World Medical Association (Declaration of Helsinki) for experiments.

\section{Statistics}

The results are presented as means $\pm \mathrm{SD}$. Statistical significance of the differences between groups was calculated according by one-way ANOVA. When 
ANOVA indicated a significant difference $(\mathrm{P}<0.05)$, the mean values were compared using Tukey's least significant difference test at $\mathrm{P}<0.05$.

1) Measurements of TRP metabolites using LC-MS.

1): background

Since 5-HT and its derivatives have their own natural fluorescence based on an internal indole structure, some of TRP metabolites can be determined by liquid chromatography with fluorescence detection. However, it is not enough to analyze the total metabolites by photometric detection because compounds under kynurenine pathway do not have fluorescenceal though only kynurenic acid (KNYA) can be detected as zinc chelate compound under zinc acetate solution.

In the past years, liquid chromatograph mass spectrometry (LC-MS) has been utilized in clinical and biological analyses. Since LC-MS with electrospray ionization (ESI) is a useful technique for the determination of metabolites of TRP with positive ionization, LC-MS is expected as a powerful tool for metabolites analysis.

In addition, a triple quadrupole tandem mass spectrometer also has an advantage for quantification as well as high sensitivity and selectivity of mass spectrometry.

The simultaneous analyses of TRP metabolites using LC-MS or LC-MS/MS methods for clinical samples such as human serum and plasma has been reported [12] [13]. In general, isotope labeled internal standards should be used for LC-MS/MS analysis to improve the accuracy of quantification although isotope labeled regents are expensive and limited availability [14] [15]. Even though less accurate, acceptable results can be obtained without internal standards for the screening purposes, LC-MS/MS techniques still has a huge advantage for total metabolites determination of TRP.

2): Regents and instrumentation.

The simultaneous analytical method developed can be adapted to major metabolites of TRP including melatonin in clinical sample.

The analytical targets of developed method are major metabolites, such as Tryptophan (TRP), L-5-Hydroxytryptophan(5-HTP), Serotonin (5-HT), Kynurenine (KYN), 5-hydroxy-tryptophol, Tryptophol, 5-Hydroxyindoleacetic acid (5-HIAA), Indole-3-acetic acid, Anthranilic acid (AA), Kynurenic acid (KYNA), Quinaldic acid, Indole-3-butyric acid, 3-Hydroxykynurenine (3-HKYN), 3-hydroxyanthranilic acid (3-HAA), Xanthurenic acid (XA), Melatonin and Qunolinic acid(QA). Each compound was purchased from major chemical regent manufacturers, such as Fujifilm-Wako chemical (Osaka, Japan) and Sigma-Aldrich (St. Louis, MO, USA).

Metabolites analysis was performed by a liquid chromatograph tandem mass spectrometer, the LCMS-8060 quadrupole mass spectrometer combined with Nexera X2 liquid chromatograph system (Shimadzu Corporation, Kyoto, Japan).

The targets are separated by reversed phase chromatography using C18 analytical column, L-Columns ODS2 (2.1 mm × $150 \mathrm{~mm}$, CERI, Tokyo, Japan) with a gradient elution. Mobile phases were $0.1 \%$ formic acid solution and acetoni- 
trile with the gradient elution by $5 \%$ concentration of acetonitrile in 3 minutes, then $5 \%$ to $95 \%$ in 6 minutes followed by $5 \%$ in 3 minutes at total flow rate 0.4 $\mathrm{mL} / \mathrm{min}$. The temperature of the column was $40^{\circ} \mathrm{C}$. Electrospray ionization (ESI) was used as mostly positive ionization with multi-reaction monitoring (MRM) detection.

Flow rate of the neutralizer and the drying gas were $2 \mathrm{~L} / \mathrm{min}$ and $10 \mathrm{~mL} / \mathrm{min}$, respectively. Temperature of desolvation line (heated capitally tube) was $250^{\circ} \mathrm{C}$. ESI interface was used at $400^{\circ} \mathrm{C}$ with $10 \mathrm{~L} / \mathrm{min}$ of heating gas flow. Each MRM transition was optimized using each standard solution. Optimized results were shown in Table 1.

All mother solution of $1 \mathrm{mg} / \mathrm{mL}$ had been stocked under $-80^{\circ} \mathrm{C}$ and standard samples for calibration curve were prepared prior to use as mixture solution by consideration of each range of measurement concentration.

3): Analysis of human plasma

Aliquot of $50 \mu \mathrm{L}$ human plasma was used for each sample analysis. The procedure including deproteinization is shown in Figure 1. The typical chromatograms of 17 major metabolites are shown in Figure 2 as standard solution and in Figure 3 as human plasma sample.

2) Measurements of plasma levels of tryptophan metabolites in health old men and patients of T2DM (Figure 4) [11].

\section{Methods}

We asked male acquaintances older than 50 years to participate in the experiments. The sample sizes and ages of participants are as follows; old men $(n=20$, age; $62.4 \pm 9.6)$ and patients of T2DM $(n=20$, age $67.0 \pm 10.4)$. We obtained an informed consent prior to conducting the protocol which had been approved by the Ethical Committee of Showa Women's University and Saiseikai main Hospital.

Drs. K. Matsuoka and K. Kato, who are internists checked their health carefully and examined their blood samples and recruited them if there were no health problems such as diabetes, hypertension or not serious diseases experienced in the past. They did not smoke in the past. We also excluded people who took drugs for dyslipidemia, hyperglycemia, or hypertension. Their disease histories were average 5 years. They were mainly treated by the controls of life styles such as foods intakes and exercises. No body used insulin for treatments. Some took metabolism promoting drugs such as metformin. We collected blood samples early morning. Participants were asked not to eat anything after 21.00 PM the previous evening. Plasma specimens were collected for assays of blood parameters. We obtained an informed consent prior to conducting the protocol which had been approved by the Ethical Committee of Showa Women's University and Saiseikai Shibuya Satellite Clinic.

Fasting plasma samples of T2DM patients were obtained early morning. Some patients used antidiabetic drugs, but most of them were treated by keeping life 
Table 1. MRM transition.

\begin{tabular}{|c|c|c|c|c|c|c|}
\hline \multirow{2}{*}{ Compound } & \multirow{2}{*}{$\begin{array}{l}\text { Molecular } \\
\text { weight }\end{array}$} & \multirow{2}{*}{$\begin{array}{l}\text { Monoisotopic } \\
\text { mass }\end{array}$} & \multicolumn{2}{|c|}{ Quantitation (m/z) } & \multicolumn{2}{|c|}{ Qualification (m/z) } \\
\hline & & & Precursor ion & Product ion & Precursor ion & Product ion \\
\hline Tryptophan & 204.225 & 204.09 & 205.1 & 188.1 & 205.1 & 146.1 \\
\hline L-5-Hydroxytryptophan & 220.225 & 220.085 & 221.1 & 204.05 & 221.1 & 162 \\
\hline Serotonin & 176.215 & 176.095 & 177.1 & 160.1 & 177.1 & 115.05 \\
\hline L-Kynurenine & 208.214 & 208.085 & 209.1 & 192 & 209.1 & 94.05 \\
\hline 5-Hydroxytryptophol & 177.2 & 177.079 & 178.1 & 160.1 & 178.1 & 115.05 \\
\hline Tryptophol & 161.2 & 161.084 & 162.1 & 144.05 & 162.1 & 117.1 \\
\hline 5-Hydroyindole-3-acetic acid & 190.176 & 190.051 & 192.1 & 146.05 & 192.1 & 110 \\
\hline Indole-3-acetic acid & 175.184 & 175.063 & 176.1 & 130.05 & 176.1 & 77.05 \\
\hline Anthranilic acid & 136.129 & 136.04 & 138.1 & 120.05 & 138.1 & 65.05 \\
\hline Kynurenic acid & 189.167 & 189.043 & 190.1 & 144.05 & 190.1 & 89.1 \\
\hline Quinaldic acid & 172.161 & 172.04 & 174.1 & 128.05 & 174.1 & 156.05 \\
\hline Indole-3-butylic acid & 202.23 & 202.087 & 204.1 & 186.1 & 204.1 & 130.1 \\
\hline 3-Hyroxykinurenine & 224.213 & 224.08 & 225.15 & 208.2 & 225.15 & 162.15 \\
\hline Hyroxyanthranilic acid & 152.128 & 152.035 & 154.15 & 136.2 & 154.15 & 80.15 \\
\hline Xanthutrenic acid & 205.167 & 205.038 & 206.15 & 160.2 & 206.15 & 132.2 \\
\hline Melatonin & 232.279 & 232.121 & 232.2 & 174.1 & 232.2 & 130.05 \\
\hline Qunolinic acid & 167.12 & 167.022 & 168 & 78.1 & 168 & 150 \\
\hline
\end{tabular}

Human plasma

$50 \mu \mathrm{L}$ in $1.5 \mathrm{~mL}$ test Tube

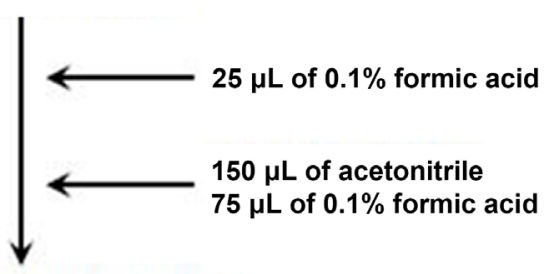

Vortexed mixing in $\mathbf{3 0}$ seconds

Leaving in 5 minutes

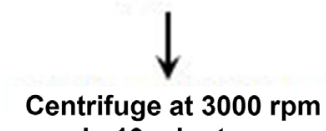

in 10 minutes.

$120 \mu \mathrm{L}$ of the supernatant

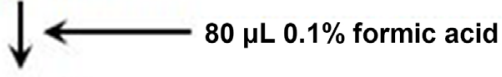

Vortexed mixing in $\mathbf{3 0}$ seconds

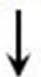

$1 \mu \mathrm{L}$ injection

Figure 1. The procedure of deproteinization. 


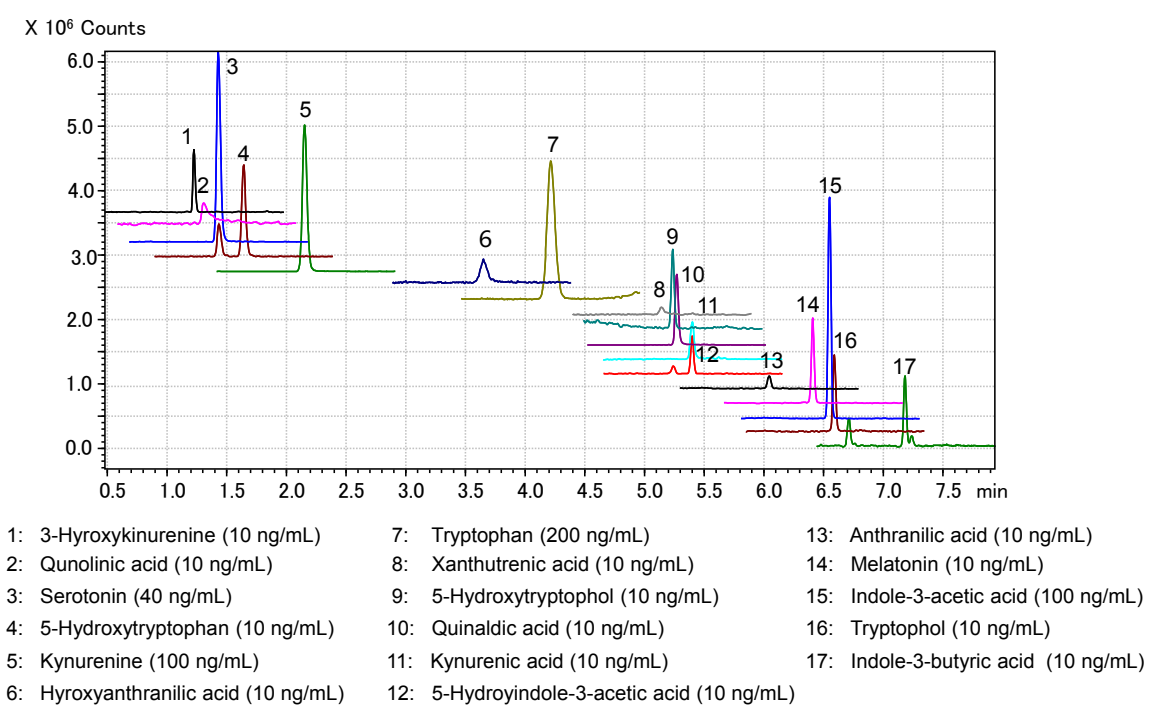

Figure 2. Chromatograms of 17 major metabolites of tryptophan.

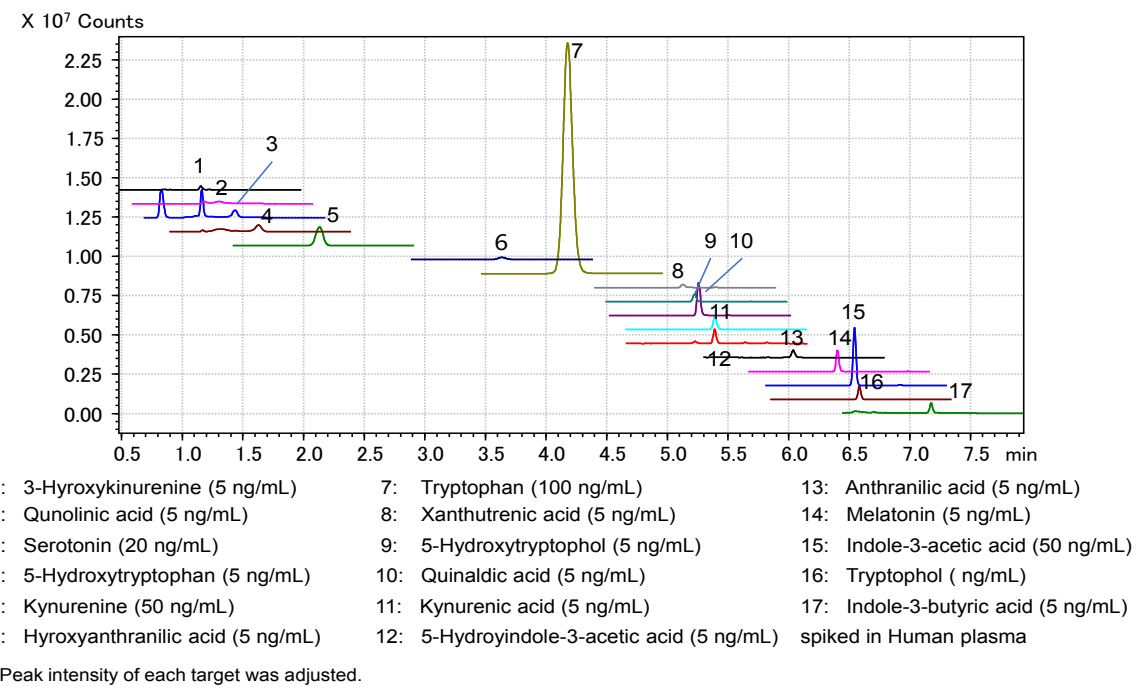

Figure 3. Chromatogram of human plasma sample.

styles such as food intakes in good condition. No patients used insulin for treatment.

As shown in Table 2, there are no statistical differences of background parameters between healthy old men and patients of T2DM.

As shown in Table 3, the plasma levels of tryptophan metabolites of T2DM patients are higher than those in old men except for kynurenine. and indole butyric acid.

Figure 5 shows that plasma levels of serotonin were higher in T2DM patients than old men.

Figure 6 shows that 5-HIAA levels were higher in old men than T2DM patients.

In contrast to data of KYN, plasma levels of KYNA were higher in T2DM patients compared to those in old menas shown in Figure 7. 


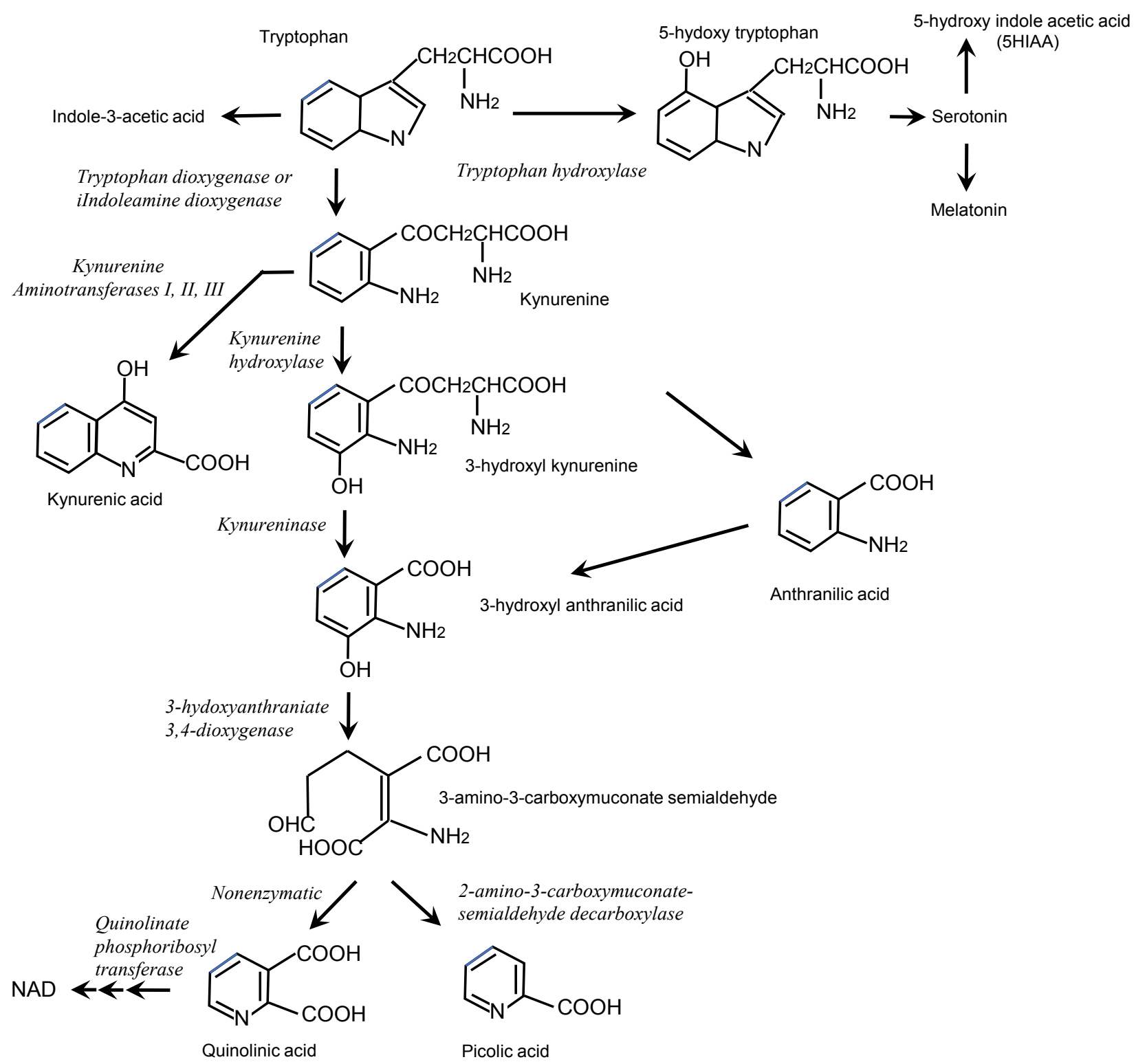

Figure 4. TRP pathways.

As shown in Figure 8, plasma levels of 3-hyroxyanthranilic acid were higher in T2DM patients than old men.

\section{Discussion}

Scientists have paid attention to TRP metabolism due to its role in the production of serotonin in the brain, which is an important transmitter regulating mood, anxiety, cognition or memory [16]. Serotonin is further converted to 5-HIAA, or N-acetylserotonin or melatonin, which controls circadian rhythmicity. However, as stated in INTRODUCTION, the majority of free TRP is degraded through the kynurenine pathway. The final product is nicotinamide adenine dinucleotide $\left(\mathrm{NAD}^{+}\right)$, which is now under investigation for therapeutic target of several diseases [17]. Kynurenine (KYN) and its metabolites are also 
Table 2. Backgrounds of participants.

\begin{tabular}{|c|c|c|c|}
\hline Subject & Healthy old men $(n=20)$ & $\mathrm{DM}(\mathrm{n}=20)$ & significance \\
\hline Age(years) & $61.5 \pm 8.8$ & $65.7 \pm 10.4$ & \\
\hline Height(m) & $1.68 \pm 0.07$ & $1.70 \pm 0.06$ & \\
\hline Weight $(\mathrm{kg})$ & $67.2 \pm 12.9$ & $68.2 \pm 11.5$ & \\
\hline $\mathrm{BMI}\left(\mathrm{kg} / \mathrm{m}^{2}\right)$ & $23.7 \pm 3.9$ & $23.7 \pm 3.7$ & \\
\hline
\end{tabular}

Table 3. TRP metabolites of old men and T2DM patients.

\begin{tabular}{cccc}
\hline Subjects & old men $(\mathrm{n}=20)$ & $\mathrm{DM}(\mathrm{n}=20)$ & significance \\
\hline Tryptophan & $1407 \pm 272$ & $1323 \pm 316$ & \\
L-5-Hydroxytryptophan & $0.014 \pm 0.029$ & $0.048 \pm 0.038$ & $\mathrm{p}<0.01$ \\
Serotonin & $0.66 \pm 1.45$ & $2.15 \pm 4.54$ & $\mathrm{p}<0.05$ \\
Kynurenine & $35.3 \pm 22.4$ & $33.8 \pm 11.2$ & \\
5-hydroxy-tryptophol & $\operatorname{Tr}$ & $\operatorname{Tr}$ & $\mathrm{Tr}$ \\
Tryptophol & $\operatorname{Tr}$ & $0.934 \pm 0.405$ & $\mathrm{p}<0.01$ \\
5-Hydroxyindoleacetic acid & $0.634 \pm 0.240$ & $54.1 \pm 28.8$ & $\mathrm{p}<0.01$ \\
Indole-3-acetic acid & $42.6 \pm 15.0$ & $0.298 \pm 0.110$ & $\mathrm{p}<0.01$ \\
Anthranilic acid & $0.220 \pm 0.165$ & $1.79 \pm 0.66$ & \\
Kynurenic acid & $1.21 \pm 0.41$ & $0.156 \pm 0.180$ & \\
Quinaldic acid & $0.153 \pm 0.134$ & $0.099 \pm 0.159$ & \\
3-Indolebutyric acid & $0.121 \pm 0.097$ & $0.105 \pm 0.053$ & \\
3-Hydroxykynurenine & $0.058 \pm 0.050$ & $0.310 \pm 0.214$ & \\
3-hydroxyanthranilic acid & $0.133 \pm 0.149$ & $0.254 \pm 0.109$ & \\
Xanthurenic acid & & $0.305 \pm 0.107$ & \\
\hline
\end{tabular}

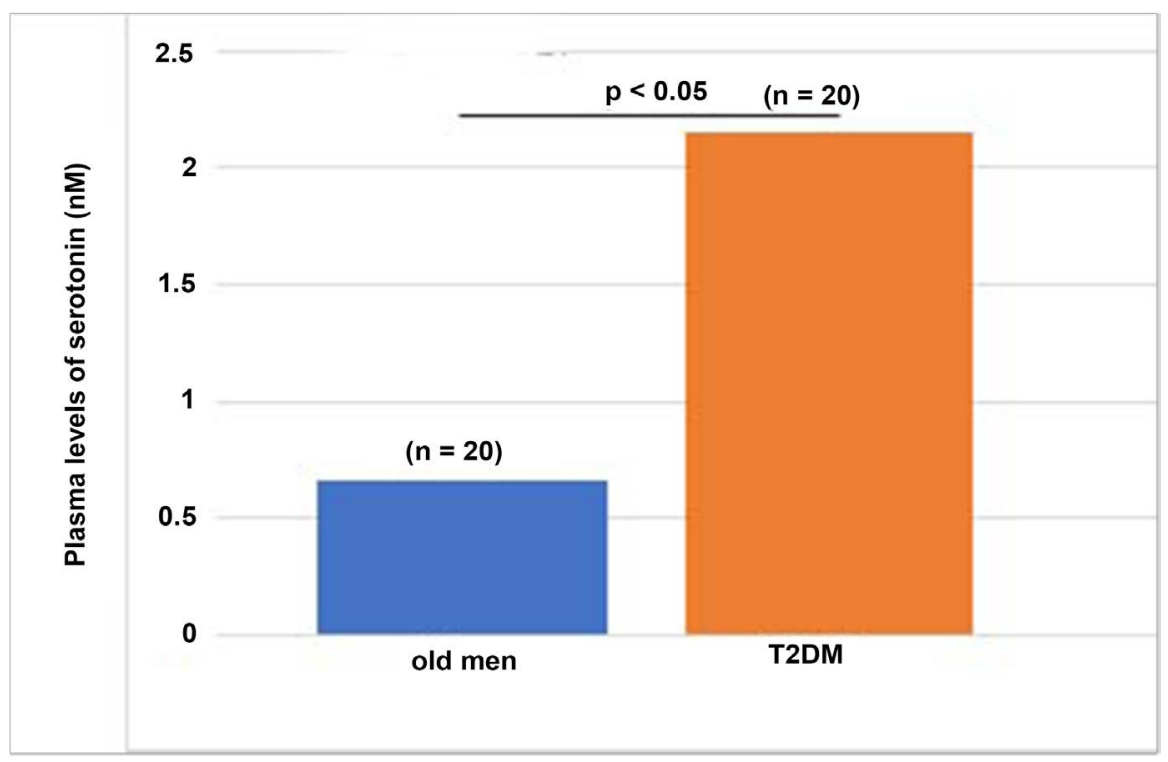

Figure 5. Serotonin levels in old men and T2DM patients. a vs. b; $\mathrm{p}<0.05$, ab vs. a or $\mathrm{b}$; not significant. 


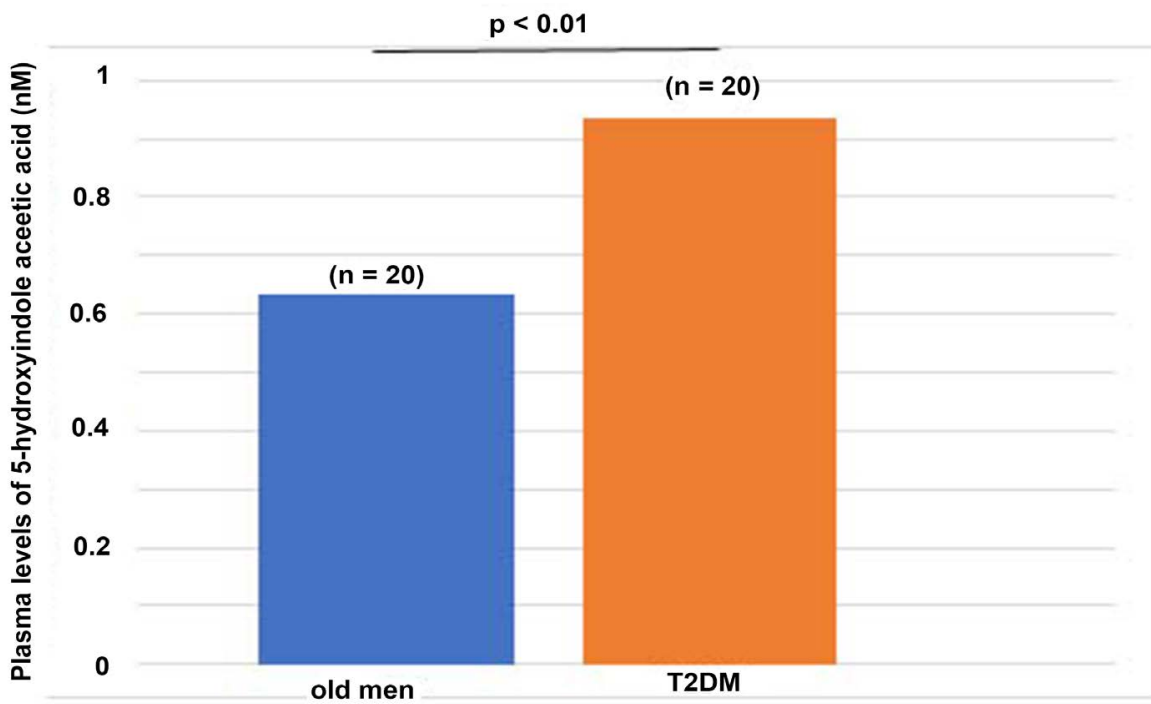

Figure 6. 5-HIAA (5-hydroxyindoleacetic acid) levels in old men and T2DM patients. $p<0.01$

$(n=20)$

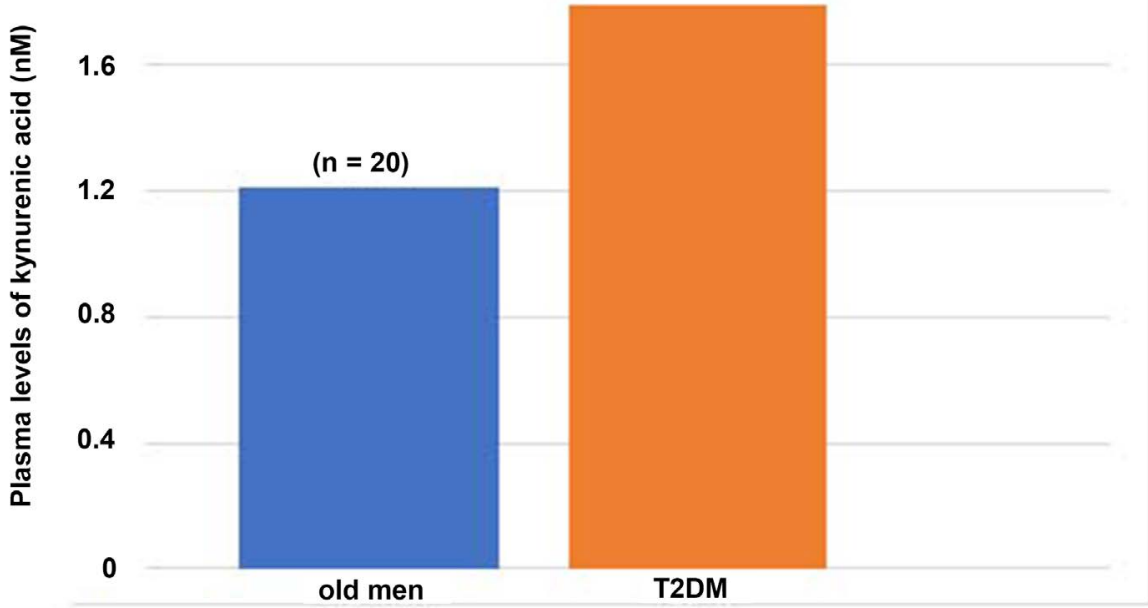

Figure 7. Plasma levels of KYNA in old men and T2DM patients.

known for their actions in the central nervous system. They stimulate or suppress the functions of glia cells. Defects in KYN signaling are shown in mouse models of Alzheimer disease or Huntington disease.

TRP is an essential amino acid which must be intaken from the food. Eggs, fish, dairy products, meat, and legumes contain higher levels of TRP compared with other foods of vegetable origin.

We first measured plasma levels of various amino acids in healthy old men and T2DM patients. Data of plasma levels of amino acids have been reported before [11].

Plasma levels of some non-essential amino acids such as glutamic acid and aspartic acid were higher in T2DM patients. On the other hand, glutamine levels were higher in healthy old men. 


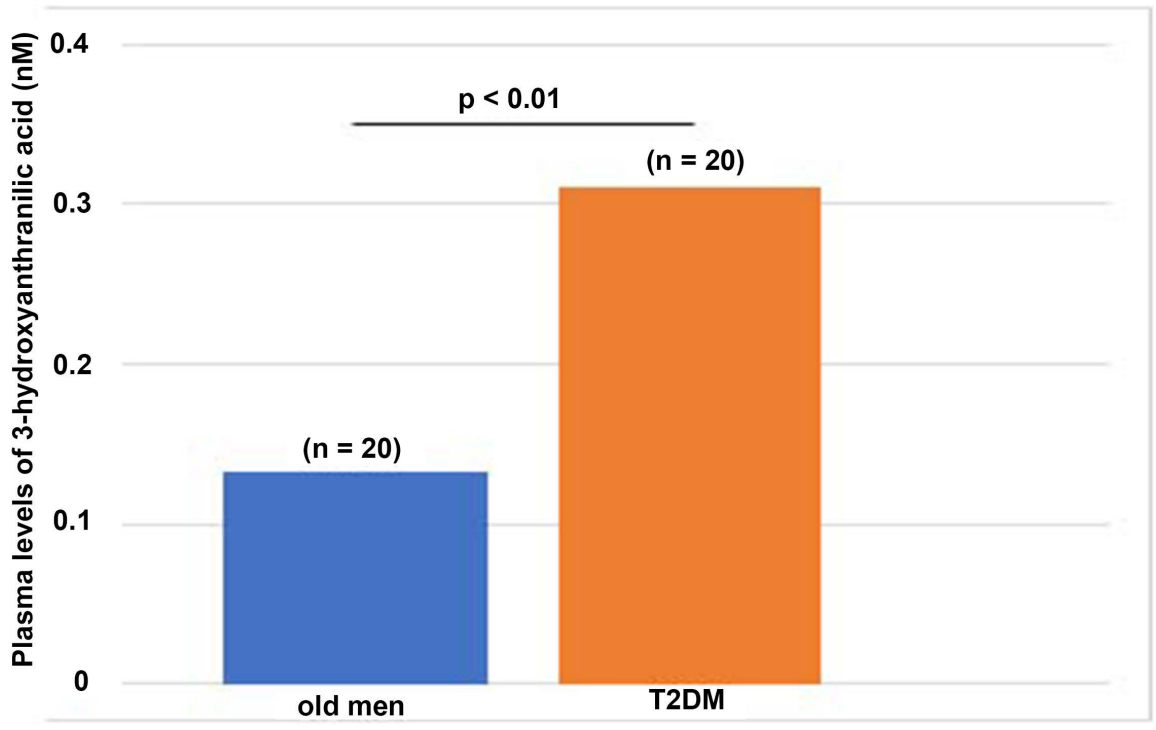

Figure 8. Plasma levels of 5-hydroxyanthranilic acid in old men and T2DM patients.

Of interest, TRP levels were lower in T2DM patients, and the levels of branched chain amino acids such as leucine, isoleucine and valine were higher in T2DM patients.

There are some papers indicating that insulin regulates the metabolism of carbohydrate, lipid, protein, and amino acid [18]. Proteolysis and the release of amino acids produced are inhibited by insulin and insulin stimulates amino acid uptake and protein synthesis in skeletal muscle [19] [20]. High insulin levels were shown to stimulate skeletal muscle protein synthesis [21]. As to individual amino acids, the plasma levels of alanine, phenylalanine, valine, leucine, isoleucine, and tyrosine were shown to increase and the plasma levels of histidine and glutamine were shown to decrease in hyperglycemia [22].

The majority of TRP is imported into the gut, where only a fraction is used. The remaining TRP is imported to the liver and then transported to tissues such as the brain, heart, and skeletal muscle.

Although TRP metabolites play important roles in the intestine, pancreas, skeletal muscle, liver or immune system, we here discuss their roles in pathogenesis of diabetes mellitus.

As stated above TRP metabolism is linked to inflammation and immune suppression. The increased production of serotonin is reported to be related to pathogenesis of diabetes [23]. Our results also show that plasma levels of serotonin were significantly increased in T2DM patients (Table 3, Figure 5). Tryptophan hydroxylase (TRP hydroxylase) which converts TRP to 5-HTP is known to be a rate-limiting factor (24) The increased plasma levels of 5-HTP and 5-HIAA may mean that serotonin was rapidly formed from TRP and also rapidly metabolized to 5-HIAA in patients of T2DM.

Chronic stress and low-grade inflammation are major risk factors in prediabetes to diabetes transition. They can change the balance of TRP metabolism 
toward KYN, 3-HYK, and KNA, both by activating TDO (tryptophan 2,3 dioxygenase)/IDO (indoleamine 2,3 dioxygenase) and by reducing the availability of pyridoxal-5-phosphate, a necessary cofactor for many KP (kynurenine pathway) enzymes.

Diabetic patients show increased levels of XA (xanthurenic acid) and KNA in urine, which have been consequently suggested as biomarkers for type 2 diabetes mellitus (T2DM) [25] [26]. Our results, however, show that although plasma levels of KNA was increased, those of XA were not elevated in patients of T2DM. Moreover, TRP metabolites inhibit both proinsulin synthesis and glucose- and leucine-induced insulin release from rat pancreatic islets, and XA in particular binds to circulating insulin and prevents its action on target cells [27]. Recently, $\mathrm{KN}$-AhR (aryl hydrocarbon receptor) signaling in mice has been suggested to play a role in the etiology of obesity.

In the present research we report that the degradation of TRP into the serotonin and kynurenine pathway was increased in T2DM patients, but that plasma levels of not all the metabolites increased in patients of T2DM.

These results, however, support the idea that there are increased immune and stress activities in diabetes. Quinolinic acid is an agonist of N-methyl-D-aspartate receptor and kynurenic acid is an antagonist [7] [8]. Our results show that the plasma levels of KNA was increased in patients of T2DM. So it is possible that the balance of nerve agonist and antagonist is impaired in diabetes.

In fact, TRP metabolites were shown to be increased in patients of acute coronary diseases. in which acute or chronic stress is implicated as causes. In patients with suspected stable angina pectoris, elevated levels of plasma kynurenines predicted the increased risk of acute myocardial infarction, and risk estimates were generally stronger in subgroups of impaired glucose homeostasis [28]. IDO activation was shown to be associated with depressive symptoms of coronary artery disease [29]. These results may suggest that stress may activate TRP degradation and cause thrombosis in the artery.

As indicated before, we applied electric foot shock to rats and measured TRP metabolites in the plasma, the central nervous system and peripheral tissues. Plasma levels of TRP increased significantly immediately after the foot shock and returned to normal values within 24 hours. TRP levels also increased in all the brain areas immediately after stress application. Foot shock elevated the levels of KYN in the plasma, liver, kidney and every part of the brain. 3-HKN and KNA levels increased in the brain. These results indicate that stress activates not only serotonergic pathway but also KYN pathway in the central nervous system and periphery. Some metabolites of KYN pathway, such as 3-HKYN are neurotoxic while other metabolite such as KNA is neuroprotective. Increase in serotonin levels in the hypothalamus and midbrain stabilizes emotion and prevents mood disorders. Therefore, some brain dysfunction resulting from stress may be prevented by the metabolites of TRP. The balance of these functions may be important in the maintenance of nerve integrity and peripheral homeostasis under stress. 
We think that the such stress may contribute to the etiology of T2DM.

We have already shown that intake of various foods (carbohydrate, lipid and protein) or BMI had little to do with various plasma factors such as amino acids, lipids, glucose, or insulin [30].

We want to know if there are any relationships between various foods intakes and plasma levels of TRP metabolites, which will be reported later.

\section{Acknowledgements}

Experiments were designed and performed by all of the authors. AT wrote a manuscript. Statistical analyses were done by FS. All authors read the manuscript and approved the final version. All the authors had responsibilities for the final content. No conflicts of interest for any author.

\section{Financial Support}

This study was supported by grants by NPO "International Projects on Food and Health."

\section{Conflicts of Interest}

The authors declare no conflicts of interest regarding the publication of this paper.

\section{References}

[1] Cervenka, I., Agudelo, L.Z. and Ruas, J.L. (2017) Kynurenines: Tryptophan's Metabolites in Exercise, Inflammation, and Mental Health. Science, 357, pii: eaaf9794. https://doi.org/10.1126/science.aaf9794

[2] Yates, C.A. and Herbert, J. (1976) Differential Circadian Rhythms in Pineal and Hypothalamic 5-HT Induced by Artificial Photoperiods or Melatonin. Nature, 262, 219-220. https://doi.org/10.1038/262219a0

[3] Stone, T.W., Stoy, N. and Darlington, L.G. (2013) An Expanding Range of Targets for Kynurenine Metabolites of Tryptophan. Trends in Pharmacological Sciences, 34, 136-143. https://doi.org/10.1016/j.tips.2012.09.006

[4] Malyszko, J., Urano, T., Yan, D., Serizawa, K., Kozima, Y., Takada, Y. and Takada, A. (1994) Foot Shock-Induced Changes in Blood and Brain Serotonin and Related Substances in Rats. The Japanese Journal of Physiology, 44, 35-47. https://doi.org/10.2170/jjphysiol.44.35

[5] Malyszko, J., Urano, T., Takada, Y. and Takada, A. (1995) Amino Acids, Serotonin, and 5-Hydroxyindoleacetic Acid Following Foot Shock in Rats. Brain Research Bulletin, 36, 137-140. https://doi.org/10.1016/0361-9230(94)00178-4

[6] Pawlak, D., Takada, Y., Urano, T. and Takada, A. (2000) Serotonergic and Kynurenic Pathways in Rats Exposed to Foot Shock. Brain Research Bulletin, 53, 197-205. https://doi.org/10.1016/S0361-9230(00)00252-5

[7] Okuno, E., Nishikawa, T. and Nakamura, M. (1996) Kynurenine Aminotransferases in the Rat. Localization and Characterization. Advances in Experimental Medicine and Biology, 398, 455-464. https://doi.org/10.1007/978-1-4613-0381-7_71

[8] Nakagami, Y., Saito, H. and Katsuki, H. (1996) 3-Hydroxykynurenine Toxicity on 
the Rat Striatum in Vivo. The Japanese Journal of Pharmacology, 2, 183-186. https://doi.org/10.1254/jjp.71.183

[9] Price, L.H., Charney, D.S., Delgado, P.L., Goodman, W.K., Krystal, J.H., Woods, S.W. and Heninger, G.R. (1990) Clinical Studies of 5-HT Function Using i.v. L-Tryptophan. Progress in Neuro-Psychopharmacology \& Biological Psychiatry, 14, 459-472.

[10] Stone, T.W. (1993) Neuropharmacology of Quinolinic and Kynurenic Acids. Pharmacological Reviews, 45, 309-379.

[11] Matsuoka, K., Kato, K., Takao, T., Ogawa, M., Ishii, Y., Shimizu, F., Masuda, J. and Takada, A. (2016) Concentrations of Various Tryptophan Metabolites Increase in Patients of Diabetes Mellitus Compared to Healthy Aged Male Adults. Diabetology International.

[12] Hervè, E., Beyne, E., Jamault, H. and Delacoux, E. (1996) Determination of Tryptophan and Its Kynurenine Pathway Metabolites in Human Serum by HighPerformance Liquid Chromatography with Simultaneous Ultraviolet and Fluorimetric Detection. Journal of Chromatography B, 675, 157-161. https://doi.org/10.1016/0378-4347(95)00341-X

[13] Marklová, E., Makovičková, H. and Krákorova, I. (2000) Screening for Defects in Tryptophan Metabolism. Journal of Chromatography A, 870, 289-293. https://doi.org/10.1016/S0021-9673(99)00973-5

[14] Zhu, W., Stevens, A.P., Dettmer, K., Gottfried, E., Hoves, S., Kreutz, M., Holler, E., Canelas, A.B., Kema, I. and Oefner, P.J. (2011) Quantitative Profiling of Tryptophan Metabolites in Serum, Urine, and Cell Culture Supernatants by Liquid Chromatography-Tandem Mass Spectrometry. Analytical and Bioanalytical Chemistry, 401, 3249-3261. https://doi.org/10.1007/s00216-011-5436-y

[15] Zhang, A., Rijal, K., Kah, S., Ravid, K. and Chitalia, V. (2017) A Mass Spectrometric Method for Quantification of Tryptophan-Derived Uremic Solutes in Human Serum. Journal of Biological Methods, 4, e75. https://doi.org/10.14440/jbm.2017.182

[16] Canli, T. and Lesch, K.P. (2001) Long Story Short: The Serotonin Transporter in Emotion Regulation and Social Cognition. Nature Neuroscience, 10, 1103-1109. https://doi.org/10.1038/nn1964

[17] Cantó, C., Menzies, K.J. and Auwerx, J. (2015) NAD ${ }^{+}$Metabolism and the Control of Energy Homeostasis: A Balancing Act between Mitochondria and the Nucleus. Cell Metabolism, 22, 31-53. https://doi.org/10.1016/j.cmet.2015.05.023

[18] Felig, P. (1975) Amino Acid Metabolism in Man. Annual Review of Biochemistry, 44, 933-955. https://doi.org/10.1146/annurev.bi.44.070175.004441

[19] Jefferson, L.S., Li, J.B. and Rannels, S.R. (1977) Regulation by Insulin of Amino Acid Release and Protein Turnover in the Perfused rat Hemicorpus. The Journal of Biological Chemistry, 252, 1476-1483.

[20] Biolo, G., Williams, B.D., Fleming, R.Y. and Wolfe, R.R. (1999) Insulin Action on Muscle Protein Kinetics and Amino Acid Transport during Recovery after Resistance Exercise. Diabetes, 48, 949-957. https://doi.org/10.2337/diabetes.48.5.949

[21] Robinson, M.M., Soop, M., Sohn, T.S., Morse, D.M., Schimke, J.M., Klaus, K.A. and Nair, K.S. (2014) High Insulin Combined with Essential Amino Acids Stimulates Skeletal Muscle Mitochondrial Protein Synthesis While Decreasing Insulin Sensitivity in Healthy Humans. The Journal of Clinical Endocrinology \& Metabolism, 99, E2574-E2583. https://doi.org/10.1210/jc.2014-2736

[22] Stancáková, A., Civelek, M., Saleem, N.K., Soininen, P., Kangas, A.J., Cederberg, H., 
Paananen, J., Pihlajamäki, J., Bonnycastle, L.L., Morken, M.A., Boehnke, M., Pajukanta, P., Lusis, A.J., Collins, F.S., Kuusisto, J., Ala-Korpela, M. and Laakso, M. (2012) Hyperglycemia and a Common Variant of GCKR Are Associated with the Levels of Eight Amino Acids in 9,369 Finnish Men. Diabetes, 61, 1895-1902. https://doi.org/10.2337/db11-1378

[23] Crane, J.D., Palanivel, R., Mottillo, E.P., Bujak, A.L., Wang, H., Ford, R.J., Collins, A., Blümer, R.M., Fullerton, M.D., Yabut, J.M., Kim, J.J., Ghia, J.E., Hamza, S.M., Morrison, K.M., Schertzer, J.D., Dyck, J.R., Khan, W.I. and Steinberg, G.R. (2015) Inhibiting Peripheral Serotonin Synthesis Reduces Obesity and Metabolic Dysfunction by Promoting Brown Adipose Tissue Thermogenesis. Nature Medicine, 21, 166-172. https://doi.org/10.1038/nm.3766

[24] Bach-Mizrachi, H., Underwood, M.D., Kassir, S.A., Bakalian, M.J., Sibille, E., Tamir, H., Mann, J.J. and Arango, V. (2006) Neuronal Tryptophan Hydroxylase mRNA Expression in the Human Dorsal and Median Raphe Nuclei: Major Depression and Suicide. Neuropsychopharmacology, 31, 814-824.

https://doi.org/10.1038/sj.npp.1300897

[25] Patterson, A.D., Bonzo, J.A., Li, F., Krausz, K.W., Eichler, G.S., Aslam, S., Tigno, X., Weinstein, J.N., Hansen, B.C., Idle, J.R. and Gonzalez, F.J. (2011) Metabolomics Reveals Attenuation of the SLC6A20 Kidney Transporter in Nonhuman Primate and Mouse Models of Type 2 Diabetes Mellitus. The Journal of Biological Chemistry, 286, 19511-19522. https://doi.org/10.1074/jbc.M111.221739

[26] Hattori, M., Kotake, Y. and Kotake, Y. (1984) Studies on the Urinary Excretion of Xanthurenic Acid in Diabetics. Acta Vitaminologica et Enzymologica, 6, 221-228.

[27] Rogers, K.S. and Evangelista, S.J. (1985) 3-Hydroxykynurenine, 3-Hydroxyanthranilic Acid, and o-Aminophenol Inhibit Leucine-Stimulated Insulin Release from Rat Pancreatic Islets. Proceedings of the Society for Experimental Biology and Medicine, 178, 275-278.

[28] Pedersen, E.R., Tuseth, N., Eussen, S.J., Ueland, P.M., Strand, E., Svingen, G.F., Midttun, Ø., Meyer, K., Mellgren, G., Ulvik, A., Nordrehaug, J.E., Nilsen, D.W. and Nygård, O. (2015) Associations of Plasma Kynurenines with Risk of Acute Myocardial Infarction in Patients with Stable Angina Pectoris. Arteriosclerosis, Thrombosis, and Vascular Biology, 35, 455-462.

https://doi.org/10.1161/ATVBAHA.114.304674

[29] Swardfager, W., Herrmann, N., Dowlati, Y., Oh, P.I., Kiss, A., Walker, S.E. and Lanctôt, K.L. (2009) Indoleamine 2,3-Dioxygenase Activation and Depressive Symptoms in Patients with Coronary Artery Disease. Psychoneuroendocrinology, 34, 1560-1566. https://doi.org/10.1016/j.psyneuen.2009.05.019

[30] Shimizu, F., Ishii, Y., Ogawa, M., Takao, T., Matsuoka, K., Kato, K. and Takada, A. (2017) Plasma Levels of Various Amino Acids and Their Changes upon Protein Uptakes in Japanese 1 Young and Old Men and Women. Integrative Food, Nutrition and Metabolism, 4, 1-5. 


\section{Abbreviations}

Tryptophan (TRP)

L-5-Hydroxytryptophan (5-HTP)

Serotonin, (5Hydroxytryptamine, 5-HT)

Kynurenine (KYN)

5-Hydroxyindoleacetic acid (5-HIAA)

Indole-3-acetic acid (IAA)

Anthranilic acid (AA)

Kynurenic acid (KYNA)

3-Hydroxykynurenine (3-HKYN)

3-hydroxyanthranilic acid (3-HAA)

Xanthurenic acid (XA)

Qunolinic acid (QA) 